(C) ACM. This is the author's version of the work. It is posted here by permission of ACM for your personal use. Not for redistribution. The definitive version was published by ACM, see http://portal.acm.org/dl.cfm. 


\title{
Watermark Detection on Quantized Transform Coefficients Using Product Bernoulli Distributions
}

\author{
Peter Meerwald and Andreas Uhl \\ Department of Computer Sciences, University of Salzburg \\ Jakob-Haringer-Str. 2, A-5020 Salzburg, Austria \\ \{pmeerw,uhl\}@cosy.sbg.ac.at
}

\begin{abstract}
Detection performance of additive spread-spectrum watermarks depends on the statistical host signal model employed to derive the detection statistic. When transform coefficients are heavily quantized, the assumption of a Cauchy or Generalized Gaussian Distribution (GGD) is hard to justify and the estimation of model parameters becomes inaccurate. In this paper we derive a Likelihood-Ratio Test (LRT) based on the product of Bernoulli distributions. The watermark detector is designed to operate on quantized (integer) transform coefficients and therefore permits straightforward integration of the watermarking scheme in popular image and video codecs. Detection performance surpasses the linear correlation detector and is competitive with the computationally more demanding LRT based on a GGD.
\end{abstract}

\section{Categories and Subject Descriptors}

I.4.10 [Image Processing and Computer Vision]: Statistical

\section{General Terms}

Algorithms, Performance, Security

\section{Keywords}

Watermarking, Spread-spectrum, Generalized Gaussian, Likelihood Ratio Test

\section{INTRODUCTION}

Watermarking has been proposed as a technology to ensure copyright protection by embedding an imperceptible, yet detectable signal in digital multimedia content such as images or video [5]. For blind watermarking, i.e. when detection is performed without reference to the unwatermarked host signal, the host interferes with the watermark signal.

Transform domains - such as the Discrete Cosine Transformation (DCT) or the Discrete Wavelet Transformation

Permission to make digital or hard copies of all or part of this work for personal or classroom use is granted without fee provided that copies are not made or distributed for profit or commercial advantage and that copies bear this notice and the full citation on the first page. To copy otherwise, to republish, to post on servers or to redistribute to lists, requires prior specific permission and/or a fee.

MM\&Sec'10, September 9-10, 2010, Roma, Italy.

Copyright 2010 ACM 978-1-4503-0286-9/10/09 ...\$10.00.
(DWT) domain - facilitate modeling human perception and permit selection of significant signal components for multimedia coding and watermark embedding. The perceptual characteristics and distributions of transform domain coefficients has been extensively studied for image compression $[2,1]$. If we assume a Gaussian host signal, it is known that the optimal detector is the straightforward linear-correlation (LC) detector [8].

Unfortunately, DCT and DWT coefficients do not obey a Gaussian law in general, which renders the LC detector suboptimal in these situations. A first approach, exploiting the fact that DCT or DWT coefficients are not Gaussian, is proposed in [7] where the authors derive an optimal detector for an additive bipolar watermark sequence in DCT transform coefficients following a Generalized Gaussian Distribution (GGD). Many approaches for optimal detection of additive watermarks embedded in transform coefficients have been proposed in literature so far $[7,13,3]$.

In this work we propose we novel watermark detector derived from on a simple model for quantized (integer) DWT or DCT coefficient values based on the bit-plane probability signatures recently introduced for texture retrieval applications $[16,4]$. The advantages of the proposed watermark detector include the reliable estimation of the model parameters even on heavily quantized data, straightforward integration of the method in multimedia codecs as the computation of the detection statistic can be implemented using integer arithmetic only, thus permitting efficient implementation. We show that detection performance surpasses the LC detector, and - in certain embedding scenarios relevant for integrated coding and watermarking - also the LRTGGD approach [7].

The remainder of the paper is structured as follows: In Section 2 we discuss the statistical model of our approach, followed by the derivation of the detection statistic in Section 3. In Section 4, we present experimental detection results and evaluate the performance of our detector under JPEG compression attacks. Section 5 concludes the paper with a discussion on open problems and an outlook on further research.

\section{MODELING QUANTIZED TRANSFORM COEFFICIENTS}

It is commonly accepted that the marginal distributions of the DWT detail subband coefficients or DCT coefficients of natural images are highly non-Gaussian but can be well modeled by the GGD $[11,2]$ or Cauchy distribution $[1,3]$. Employing the parametrization of [12], the PDF of the GGD 
with scale parameter $a>0$ and shape parameter $c>0$ is

$$
\mathrm{p}(x ; a, c)=\frac{c}{2 a \Gamma(1 / c)} \exp \left(-\left|\frac{x}{a}\right|^{c}\right)
$$

with $-\infty<x<\infty$. In contrast to the Gaussian distribution (which arises as a special case of the GGD for $c=2$ ), the GGD is a leptokurtic distribution which allows heavy-tails.

The distribution of the transform coefficients is symmetric around zero [11], hence it can be characterized by the histogram of absolute values of the subband coefficients [16]. In case the coefficients have been quantized to integer values, an absolute coefficient $|x|$ of a particular signal can be represented by

$$
|x|=\sum_{i=1}^{B} 2^{i} X_{i}
$$

where $B$ is the number of bit planes and $X_{i} \in\{0,1\}$ is a random variables representing the binary value in plane $i$.

The joint probability distribution of an absolute quantized transform coefficient is

$$
P(|x|)=P\left(X_{1}=x_{1}, X_{2}=x_{2}, \ldots, X_{B}=x_{B}\right)
$$

for $|x|=0,1, \ldots, 2^{B}-1$. Assuming statistical independence and denoting the model parameters by $p_{i}=P\left(X_{i}=1\right)$, the joint distribution of Eq. (3) can be written as a product of Bernoulli distributions (PBD) [16, 4]

$$
P\left(X_{1}=x_{1}, \ldots, X_{B}=x_{B}\right)=\prod_{i=1}^{B} p_{i}^{x_{i}}\left(1-p_{i}\right)^{1-x_{i}}
$$

characterized by the bit plane probabilities $p_{i}$.

\subsection{Model Parameter Estimation}

Following Choy and Tong [4], the maximum likelihood (ML) estimator of the model parameter $\theta=\left(p_{1}, \ldots, p_{B}\right)$ for a signal $\mathbf{x}=x[k], 1 \leq k \leq N$ of independent, absolute quantized coefficients is obtained by solving

$$
\hat{\theta}=\arg \max _{\theta} L(\mathbf{x} ; \theta) \quad \frac{\partial L(\mathbf{x} ; \theta)}{\partial p_{i}}=0
$$

where

$$
L(\mathbf{x} ; \theta)=\log \prod_{k=1}^{N} \prod_{i=1}^{B} p_{i}^{x_{i}[k]}\left(1-p_{i}\right)^{1-x_{i}[k]}
$$

is the log-likelihood function of signal $\mathbf{x}$. The probability of the occurrence of a 1 bit in bit plane $i$ is equivalent to the ML estimator, hence

$$
\hat{p}_{i}=\frac{1}{N} \sum_{k=1}^{N} x_{i}[k]
$$

In Fig. 1 we plot histograms of quantized DWT $\mathrm{HL}_{3}$ subband coefficients as well as DCT AC $(1,1)$ coefficients obtained from the luminance band of the first image of the UCID collection [17] for different quantization step sizes $\Delta$. Both, the Generalized Gaussian and the Product Bernoulli model approximate the data very well, yet parameter estimation for the PBD is less involved compared to the computational effort to obtain the GGD ML parameter estimates which requires an iterative Newton-Raphson algorithm [6]. In case of the $\mathrm{PBD}$, positive and negative coefficients were modeled separately. Different measures for the Goodness-ofFit of the PBD relative to the GGD are provided in [16] and demonstrate satisfactory approximation of the data. In [4], the authors point out two weaknesses of the GGD model: first, the GGD estimators may not exist for heavily quantized signals of small length, and second, the GGD model can only represent histogram bin counts that are monotonically decreasing towards the tails.

The straightforward Product Bernoulli model for quantized transform coefficients prompts for the derivation of an efficient LRT following the steps outlined in [7] which we undertake in the next section.

\section{EMBEDDING AND DETECTION}

We consider watermark embedding and detection in quantized transform coefficients. Let $\mathbf{f}$ denote a vector of transform coefficients $f[k]$ of length $N, k=1, \ldots, N$, obtained e.g. from a DWT subband or a DCT domain AC coefficient sub-signal, then the quantized, integer coefficients are given by $x[k]=\operatorname{round}(f[k] / \Delta)$. Additive spread-spectrum watermark embedding can be written

$$
y[k]=x[k]+w[k]
$$

where $\mathbf{w}$ denotes a pseudo-random, bipolar watermark vector with equiprobable components $w[k] \in\{-1,1\}$ generated using a secret key $K$ identifying the copyright owner. Control over the watermark embedding strength is limited in the quantized domain because a minimum of $+1 /-1$ has to be added and might lead to a perceptually noticeable difference. As a countermeasure, only certain embedding location might be selected by the embedder. The quantization step size $\Delta$ also controls the strength of the watermark signal.

We state the watermark detection problem as a hypothesis test

$$
\begin{array}{lll}
\mathcal{H}_{0}: & y[k]=x[k] & k=1, \ldots, N \\
\mathcal{H}_{1}: & y[k]=x[k]+w[k] & k=1, \ldots, N
\end{array}
$$

where $\mathcal{H}_{0}$ is termed the null hypothesis (no $\mathbf{w}=0$, or other watermark $\left.\mathbf{w}^{\prime} \neq \mathbf{w}\right)$ and $\mathcal{H}_{1}$ denotes the alternative hypothesis (watermarked with w). Considering the NeymanPearson (NP) approach to signal detection, the detector which maximizes the probability of detection for a given probability of false-alarm (i.e. deciding $\mathcal{H}_{1}$ though $\mathcal{H}_{0}$ is true) is the (log) Likelihood-Ratio Test (LRT), given by

$$
L(\mathbf{y}):=\log \frac{\mathrm{p}\left(\mathbf{y} \mid \mathcal{H}_{1}\right)}{\mathrm{p}\left(\mathbf{y} \mid \mathcal{H}_{0}\right)}>\log (\tau)=: T
$$

where $\mathrm{p}\left(\mathbf{y} \mid \mathcal{H}_{0}\right)$ denotes the PDF under $\mathcal{H}_{0}, \mathrm{p}\left(\mathbf{y} \mid \mathcal{H}_{1}\right)$ denotes the PDF under $\mathcal{H}_{1}$ and $T$ is the detection threshold. Conditioned on the host signal noise models from Section 2, the LRT statistic for the Generalized Gaussian model [7] is

$$
L_{\mathrm{GGD}}(\mathbf{y})=\frac{1}{a^{c}} \sum_{k=1}^{N}\left(|y[k]|^{c}-|y[k]-w[k]|^{c}\right)
$$

and, inserting the Product Bernoulli model, we obtain

$$
L_{\mathrm{PBD}}(\mathbf{y})=\log \frac{\prod_{k=1}^{N} \prod_{i=1}^{B} p_{i}^{|y[k]-w[k]|_{i}}\left(1-p_{i}\right)^{1-|y[k]-w[k]|_{i}}}{\prod_{k=1}^{N} \prod_{i=1}^{B} p_{i}^{|y[k]|_{i}}\left(1-p_{i}\right)^{1-|y[k]|_{i}}}
$$

which by defining $\mathbf{z}:=\mathbf{y}-\mathbf{w}$ and $p_{i}^{\prime}:=1-p_{i}$ can be rewritten 


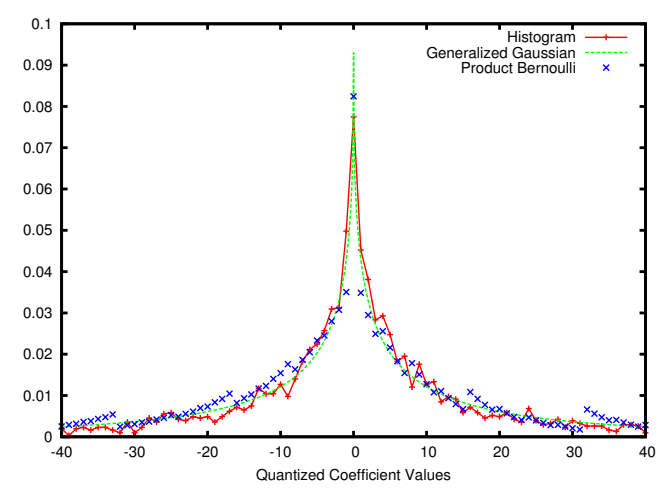

(a) DWT $\mathrm{HL}_{3}$ subband, $\Delta=1$

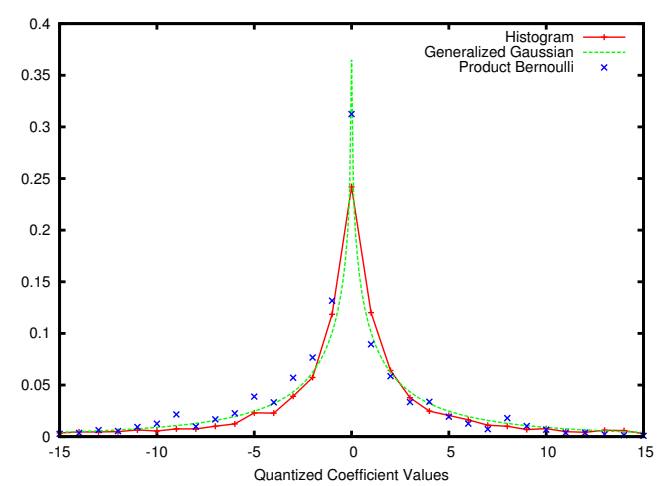

(b) DWT HL 3 subband, $\Delta=5$

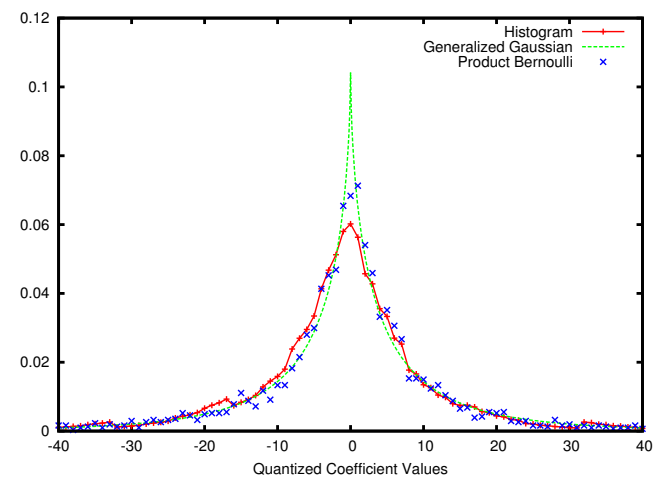

(c) $\operatorname{DCT} \mathrm{AC}(1,1), \Delta=5$

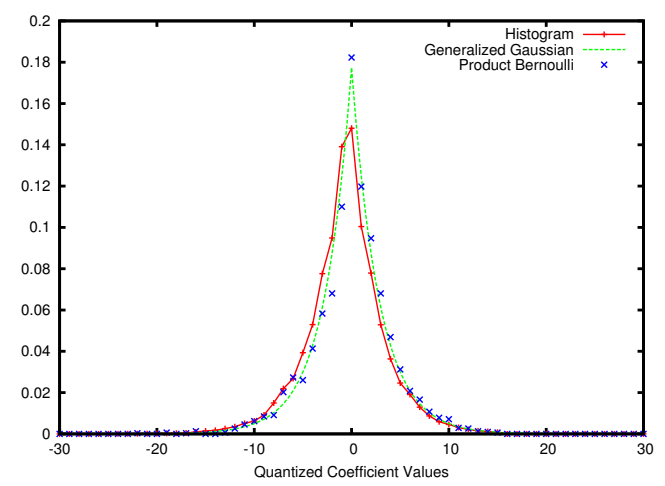

(d) $\mathrm{DCT} \operatorname{AC}(1,1), \Delta=25$

Figure 1: Histograms of quantized coefficients (w.r.t. $\Delta$ ) with Product Bernoulli and Generalized Gaussian fits. as

$$
\begin{aligned}
& L_{\mathrm{PBD}}(\mathbf{y})=\sum_{i=1}^{B}\left[\log p_{i} \sum_{k=1}^{N}|z[k]|_{i}+\log p_{i}^{\prime} \sum_{k=1}^{N}\left(1-|z[k]|_{i}\right)\right. \\
& \left.-\log p_{i} \sum_{k=1}^{N}|y[k]|_{i}-\log p_{i}^{\prime} \sum_{k=1}^{N}\left(1-|y[k]|_{i}\right)\right] .
\end{aligned}
$$

Remember that $|z[k]|_{i}$ and $|y[k]|_{i}$ denote the bit value in the $i$ th bit plane of the respective coefficient. Hence, the LRTPBD detection statistic is computed by simply summing up the $N$ bit values for each of the $B$ bit planes of the received signal, the logarithms have to be computed only once for each bit plane.

The detection statistics of Eqs. (11) and (12) as well as for the linear correlator follow a Gaussian distribution under both $\mathcal{H}_{0}$ and $\mathcal{H}_{1}$. Given that $\mu_{\mathcal{H}_{0}}$ and $\sigma_{\mathcal{H}_{0}}^{2}$ denote the mean and variance of the detection statistic under $\mathcal{H}_{0}$ and $\mu_{\mathcal{H}_{1}}$ and $\sigma_{\mathcal{H}_{1}}^{2}$ denote the mean and variance under the alternative hypothesis, the probability of false alarm (denoted by $P_{f}$ ) can be formulated as

$$
P_{f}=\mathbb{P}\left(L(\boldsymbol{y})>T \mid \mathcal{H}_{0}\right)=1 / 2 \operatorname{erfc}\left(T-\mu_{\mathcal{H}_{0}} / \sqrt{2} \sigma_{\mathcal{H}_{0}}\right)
$$

which allows to set $T$ according to the NP criterion

$$
T=\sqrt{2} \sigma \operatorname{erfc}^{-1}\left(2 P_{f}\right)+\mu_{\mathcal{H}_{0}} .
$$

The probability of missing the watermark (denoted $P_{m}$ ) is defined as the probability that $L(\mathbf{y})$ is smaller than $T$ although $H_{1}$ is true,

$$
P_{m}=\mathbb{P}\left(L\left(\mathbf{y}<T \mid \mathcal{H}_{1}\right)=1 / 2 \operatorname{erfc}\left(\mu_{\mathcal{H}_{1}}-T / \sqrt{2} \sigma_{\mathcal{H}_{1}}\right) .\right.
$$

For the performance evaluation of the LRT-GGD, LRT-PBD and the LC watermark detector, we resort to experimental estimation of the detection statistics' parameters $\mu, \sigma^{2}$ under both hypothesis using a large number of pseudo-randomly generated watermarks and detection experiments, assuming that $L(\mathbf{y})$ follows a Gaussian law.

\subsection{Application Considerations}

The proposed watermark embedding and detection method operates on quantized (integer) transform coefficients and therefore can be easily integrated in image and video codecs such as JPEG, JPEG2000 or H.264; for example, it can be plugged into the framework for robust H.264 video watermarking put forward by Noorkami et al. [14]. From a coding efficiency point-of-view, embedding in coefficients quantized to zero should be avoided as runs of consecutive zero coefficients can be coded most efficiently. This forces the watermark embedder to adaptively select transform coefficients. In a later work, Noorkami et al. [15] have coined the term Location Unaware Detection (LUD) for the scenario where the embedder decides upon the embedding location and the detector has only partial information (e.g. via a perceptual model) where the watermark is hidden.

Noorkami et al. [14, 15] recognize that the GGD is an adequate model for quantized $4 \times 4$ DCT residual coefficients, nevertheless a sub-optimal LC detector is used. Similarly, Wang et al. [18] find that the Cauchy distribution is a good match for quantized H.264 DCT coefficients, yet an ad-hoc sign detector is used. Our own experiments showed that GGD and Cauchy model parameters are hard to reliably estimate on heavily quantized data (e.g. H.264 $4 \times 4$ DCT residuals) and that the corresponding LRT detectors 


\begin{tabular}{ccc}
\hline PSNR $(\mathrm{dB}) / \sigma$ & $\Delta=5$ & $\Delta=10$ \\
\hline \hline all & $49.22 / 0.02$ & $44.76 / 0.04$ \\
non-zero & $49.53 / 0.18$ & $45.69 / 0.52$ \\
\hline
\end{tabular}

Table 1: Average PSNR (dB) and standard deviation when embedding in all and non-zero quantized DWT coefficients (w.r.t. $\Delta$ ) of the UCID images' luminance component.

do not achieve optimal performance even employing ML estimates [10].

The next section provides detection performance comparisons, also considering the LUD scenario where the watermark is embedded in non-zero quantized coefficients only.

\section{EXPERIMENTAL RESULTS}

Experimental detection results in this paper are obtained on the 1338 color images $(512 \times 384$ pixels $)$ of the UCID image database [17]. For each image, 1000 randomly generated, bipolar watermarks are embedded to allow estimation of the detection statistics' parameters. Using Eqs. (15) and (16), the detection threshold and experimental probability of miss can be determined. The watermark is embedded in the 3072 wavelet coefficients of the quantized $\mathrm{HL}_{3}$ subband of the luminance component obtained by DWT decomposition using the biorthogonal $\operatorname{CDF} 7 / 9$ filter, $\Delta$ indicates the quantization step size, no perceptual shaping is performed. Source code and scripts to reproduce results will become available at http://www.wavelab.at/sources.

Table 1 lists the average PSNR (dB) and its standard deviation for the watermarked luminance component of the UCID images obtained for two different embedding scenarios.

In Fig. 2 the experimental probability of miss $P_{m}$ given a false-alarm rate of $P_{f}=10^{-3}$ is shown for the linear correlation (LC), the LRT-GGD and the proposed LRT-PBD detector for the case that the watermark is embedded in all quantized subband coefficients. We have arranged the experimental $P_{m}$ determined for each image in ascending order for each detector with the intention to allow a visual comparison over a large set of images. Figures 2(a) and 2(b) distinguish between quantization step size $\Delta=5$ and $\Delta=10$. As expected, the probability of miss is lower for a larger quantization step size as the power of the watermark becomes stronger relative to the host signal. LRT-PBD and LRT-GGD clearly outperform the linear correlation detector, the LRT-GGD achieves the best detection performance.

Figure 3 considers the case where the watermark is embedded only in non-zero quantized subband coefficients. This is the scenario of a bit-rate aware watermark embedder which might be integrated in an image or video codec. The idea is to preserve zero-quantized coefficients as modifying these coefficients has a negative impact on coding bit rate as well as perceptual fidelity. This embedding strategy poses a challenge for the detector as the precise set of watermarked coefficients is unknown, cf. Location-Unaware Detection (LUD) [15]. Detection performance decreases relative to the previous embedding scenario as fewer coefficients are watermarked. LRT-PBD and LRT-GGD still perform better than the linear correlation detector, but now the LRT-PBD obtains better results than the LRT-GGD detector. The LRT-

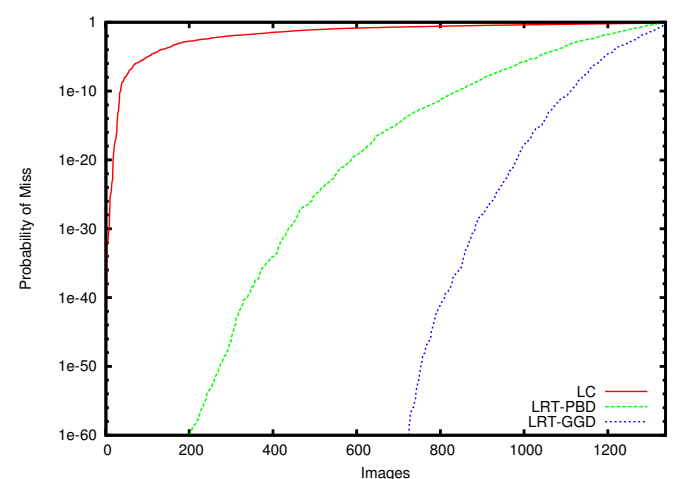

(a) $\Delta=5$

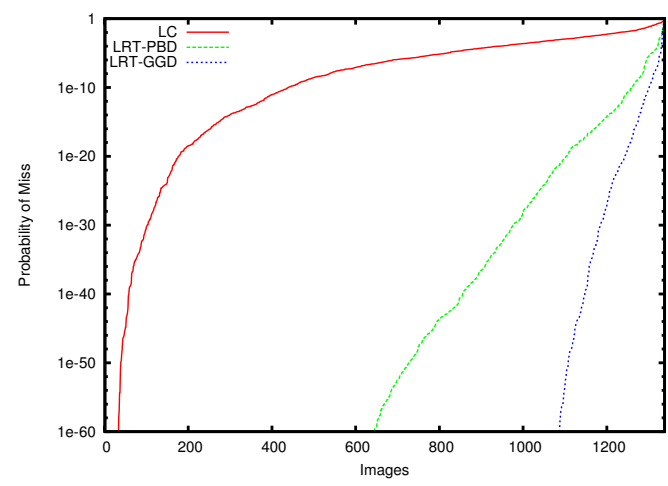

(b) $\Delta=10$

Figure 2: Probability of Miss $\left(P_{m}\right)$ of the LC, LRTPBD and LRT-GGD detectors when embedding in all quantized $\mathrm{HL}_{3}$ subband coefficients $(\Delta=5$ and $\Delta=10)$ for the UCID images $\left(P_{f}=10^{-3}\right)$.

PBD detection statistic permits to analyze the contribution of each bit plane towards detection performance: we observe only a marginal impact of the first and highest bit planes on the detector.

Detection performance under JPEG compression attack $(Q=70)$ is plotted in Fig. 4 when embedding in all and in non-zero quantized coefficients only. In the first case, the LC detector is clearly outperformed while in the LUD detection scenario, the advantage of the LRT-GGD and LRT-PBD detector diminishes. The LRT-PBD detector achieves slightly better performance than the LRT-GGD detector when embedding in non-zero coefficients only.

As an improvement for the LRT-PBD detector, positive and negative quantized coefficients can be modeled separately employing two sets of model parameters, $p_{i}^{+}$and $p_{i}^{-}$; then, instead of taking the absolute value in Eq. (13), separate summation over positive and negative $z[k]$ and $y[k]$ has to be implemented.

\subsection{Computational Analysis}

Computation of the LRT-PBD detection statistic is carried out by counting the occurrence of 1 bits for each bitplane $i(1 \leq i \leq B, B \leq 8$ typically) which can be implemented by summing up the result of a binary and (masking) and a bit shift operation for each coefficient. On the other hand, the LRT-GGD detection statistic requires two floating-point exponentiations per coefficient and accumula- 


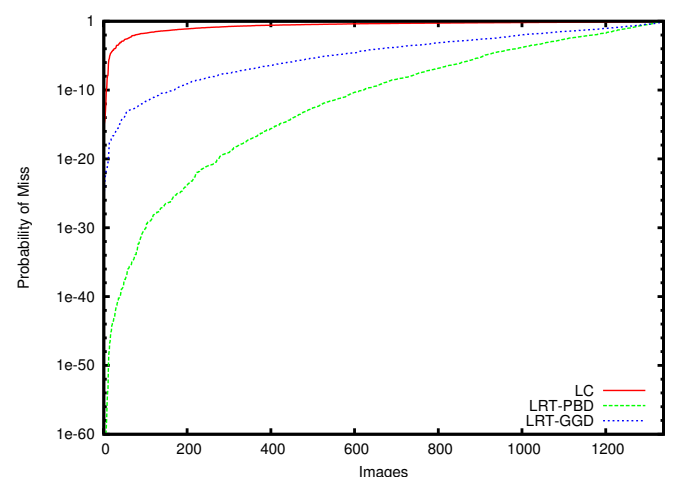

(a) $\Delta=5$

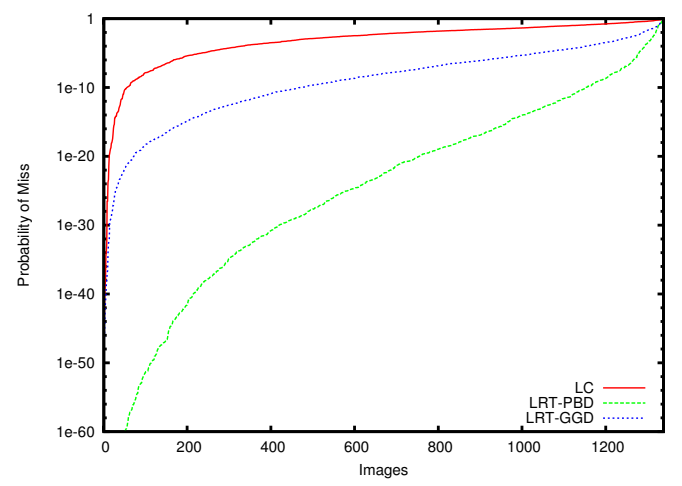

(b) $\Delta=10$

Figure 3: Probability of Miss $\left(P_{m}\right)$ of the LC, LRT-PBD and LRT-GGD detector when embedding in the non-zero quantized $\mathrm{HL}_{3}$ subband coefficients $(\Delta=5$ and $\Delta=10)$ for the UCID images $\left(P_{f}=10^{-3}\right)$.

\begin{tabular}{cccc}
\hline Detector & LC & LRT-PBD & LRT-GGD \\
\hline \hline Runtime $(\mathrm{ms})$ & 1.47 & 16.42 & 221.90 \\
\hline
\end{tabular}

Table 2: Average runtime (Intel Core2 $2.6 \mathrm{GHz}$ CPU) in milliseconds for a signal of length $N=$ 1000000.

tion of the result. On contemporary CPUs, bit operations execute within one clock cycle while floating-point exponentiation requires several hundred cycles.

Likewise, for model parameter estimation simple counting of 1 bits is necessary to establish the parameters $p_{i}$ of the Product Bernoulli distribution. For the Generalized Gaussian distribution, maximum likelihood estimation of the parameters requires to carry out an iterative Newton-Raphson algorithm [6], even though fast, approximative methods [9] are available.

The average runtime in milliseconds on an Intel Core2 $2.6 \mathrm{GHz} \mathrm{CPU}$ for the computation of the detection statistics over a signal of length $N=1000000$ in shown in Table $2 ; B=8$ for the LRT-PBD detector. The $\mathrm{C}++$ code was compiled with GCC 4.2 using -02 -march=native. The $\mathrm{LC}$ detector is clearly the fastest, followed by the proposed LRT-PBD scheme. Runtime of LRT-GGD detector is dominated by the floating-point exponentiation operation, improvements can be expected using SIMD instructions (e.g.

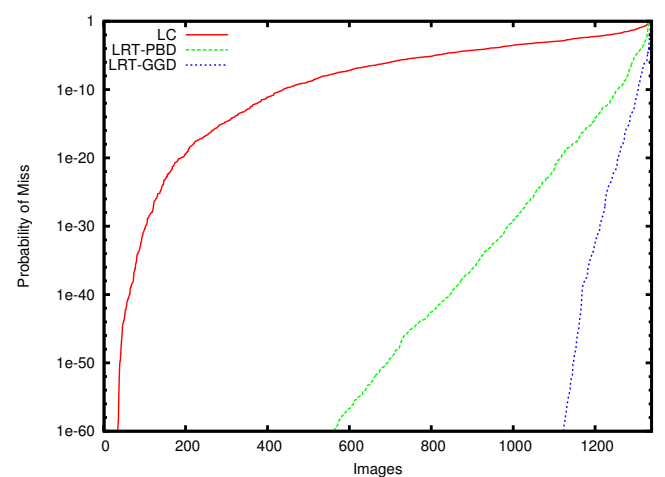

(a) embedding in all coefficients

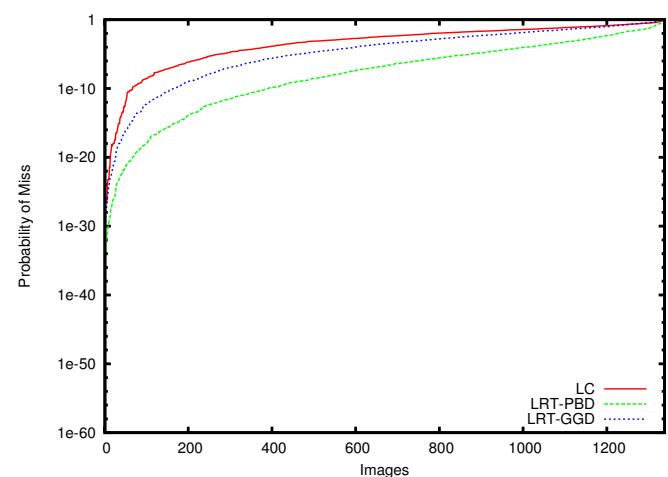

(b) embedding in non-zero coefficients

Figure 4: Probability of Miss $\left(P_{m}\right)$ of the LC, LRTPBD and LRT-GGD detector under JPEG compression $(Q=70)$ when embedding in all and non-zero quantized $\mathrm{HL}_{3}$ subband coefficients $(\Delta=10)$ for the UCID images $\left(P_{f}=10^{-3}\right)$.

SSE2 on the Intel platform) and an approximative implementation of the pow() function. The runtime of the LRTPBD detector could be improved by skipping processing of bit planes $i=1$ and $i>5$ as they only marginally contribute to the detection performance.

\section{CONCLUSION}

We have proposed a LRT watermark detector based on the Product Bernoulli model for quantized, integer transform coefficients which provides a good trade-off between computational efficiency and detection performance. The watermarking method fits in a framework for robust watermarking integrated in H.264 [14] and should improve the detection performance over the LC detector, especially in the LUD scenario where the encoder adaptively selects the embedding locations. Additional work is required to confirm the applicability for video coding and watermark detection on $4 \times 4$ DCT residual coefficients.

\section{Acknowledgments}

Supported by Austrian Science Fund (FWF) project P19159N13. 


\section{REFERENCES}

[1] Y. Altunbasak and N. Kamaci. An analysis of the DCT coefficient distribution with the H.264 video coder. In Proceedings of the IEEE International Conference on Acoustics, Speech and Signal Processing, ICASSP '04, volume 3, pages 177-180, Montreal, Canada, May 2004. IEEE.

[2] K. A. Birney and T. R. Fischer. On the modeling of DCT and subband image data for compression. IEEE Transactions on Image Processing, 4(2):186-193, Feb. 1995.

[3] A. Briassouli, P. Tsakalides, and A. Stouraitis. Hidden messages in heavy-tails: DCT-domain watermark detection using alpha-stable models. IEEE Transactions on Multimedia, 7(4):700-715, Aug. 2005.

[4] S. K. Choy and C. S. Tong. Statistical properties of bit-plane probability model and its application in supervised texture classification. IEEE Transactions on Image Processing, 17(8):1399-1405, Aug. 2008.

[5] I. J. Cox, M. L. Miller, J. A. Bloom, J. Fridrich, and T. Kalker. Digital Watermarking and Steganography. Morgan Kaufmann, 2007.

[6] M. Do and M. Vetterli. Wavelet-based texture retrieval using Generalized Gaussian density and Kullback-Leibler distance. IEEE Transactions on Image Processing, 11(2):146-158, Feb. 2002.

[7] J. R. Hernández, M. Amado, and F. Pérez-González. DCT-domain watermarking techniques for still images: Detector performance analysis and a new structure. IEEE Transactions on Image Processing, 9(1):55-68, Jan. 2000.

[8] S. M. Kay. Fundamentals of Statistical Signal Processing: Detection Theory, volume 2. Prentice-Hall, 1998.

[9] R. Krupinski and J. Purczynski. Approximated fast estimator for the shape parameter of Generalized Gaussian distribution. Signal Processing, 86(2):205-211, Feb. 2006.
[10] R. Kwitt, P. Meerwald, and A. Uhl. Efficient detection of additive watermarking in the DWT-domain. In Proceedings of the 17th European Signal Processing Conference, EUSIPCO '09, pages 2072-2076, Glasgow, UK, Aug. 2009. EURASIP.

[11] S. Mallat. Multifrequency channel decompositions of images and wavelet models. IEEE Transactions on Acoustics, Speech and Signal Processing, 37(12):2091-2110, Dec. 1989.

[12] S. Nadarajah. A generalized normal distribution. Journal of Applied Statistics, 32:685-694, Sept. 2005.

[13] A. Nikolaidis and I. Pitas. Asymptotically optimal detection for additive watermarking in the DCT and DWT domains. IEEE Transactions on Image Processing, 12(5):563-571, May 2003.

[14] M. Noorkami and R. M. Mersereau. A framework for robust watermarking of H.264 encoded video with controllable detection performance. IEEE Transactions on Information Forensics and Security, 2(1):14-23, Mar. 2007.

[15] M. Noorkami and R. M. Mersereau. Digital video watermarking in P-frames with controlled video bit-rate increase. IEEE Transactions on Information Forensics and Security, 3(3):441-455, Sept. 2008.

[16] M. H. Pi, C. S. Tong, S. K. Choy, and H. Zhang. A fast and effective model for wavelet subband histograms and its application in texture image retrieval. IEEE Transactions on Image Processing, 15(10):3078-3088, Oct. 2006.

[17] G. Schaefer and M. Stich. UCID - an uncompressed colour image database. In Proceedings of SPIE, Storage and Retrieval Methods and Applications for Multimedia, volume 5307, pages 472-480, San Jose, CA, USA, Jan. 2004. SPIE.

[18] M. Wang, K. Fan, B. Yue, and X. Yue. A content protection scheme for H.264-based video sequence. In Proceedings of the 8th ACIS International Conference on Software Engineering, Artificial Intelligence, Networking, and Parallel/Distributed Computing, SNPD '07, pages 388-393, July 2007. 